\title{
Demographic and interaction lags govern the pace and outcome of plant community responses to 21st century climate change
}

\author{
Sebastian Block ${ }^{1}$, Marc-Jacques Maechler ${ }^{2}$, Jake Alexander ${ }^{3}$, Loïc Pellissier ${ }^{4}$, and Jonathan \\ Levine $^{5}$ \\ ${ }^{1}$ Princeton University \\ ${ }^{2}$ ETH Zurich Institute of Integrative Biology \\ ${ }^{3} \mathrm{ETH}$ Zürich \\ ${ }^{4}$ ETH Zurich \\ ${ }^{5}$ Princeton Environmental Institute
}

January 27, 2022

\begin{abstract}
Forecasting the trajectories of species assemblages in response to ongoing climate change requires quantifying the time lags in the demographic and ecological processes through which climate impacts species' abundances. Since experimental climate manipulations are typically abrupt, the observed species responses may not match their responses to gradual climate change. We addressed this problem by transplanting alpine grassland turfs to lower elevations, recording species' demographic responses to climate and competition, and using these data to parameterize community dynamics models forced by scenarios of gradual climate change. We found that shifts in community structure following an abrupt climate manipulation were not simply accelerated versions of shifts expected under gradual warming, especially when they missed the rise of species benefiting from moderate warming. Time lags in demography and species interactions controlled the pace and trajectory of changing species' abundances under simulated 21st century climate change, and thereby prevented immediate diversity loss.
\end{abstract}

\section{Hosted file}

Block_et-al_Main-body_1st-submission.pdf available at https://authorea.com/users/457624/ articles/554349-demographic-and-interaction-lags-govern-the-pace-and-outcome-of-plantcommunity-responses-to-21st-century-climate-change 